\title{
Long noncoding RNA LINC00968 inhibits lung adenocarcinoma progression through sponging miR-22-5p and further restoring CDC14A
}

\section{Chao Wu}

Xi'an international Medical center hospital

\section{Xuzhao Bian}

Xi'an International Medical Center Hospital

Liyuan Zhang

Xi'an International Medical Center Hospital

Yang Wu

Xi'an International Center Hospital

Tianli Pei

Xi'an International Center Hospital

Xinpeng Han ( I Iz921114@163.com )

Northwest University

\section{Research}

Keywords: lung adenocarcinoma proliferation; LINC00968; miR-22-5p; CDC14A

Posted Date: May 22nd, 2020

DOI: https://doi.org/10.21203/rs.3.rs-30040/v1

License: (c) (i) This work is licensed under a Creative Commons Attribution 4.0 International License. Read Full License 


\section{Abstract}

Background: Lung adenocarcinoma (LUAD) is a high aggressive human cancer which usually diagnosed at advanced stages. Accumulating evidences indicate that long noncoding RNAs (IncRNAs) are crucial participants in LUAD progression.

Methods: The mRNA levels of LINC00968, miR-22-5p and cell division cycle 14A (CDC14A) were measured using quantitative real-time PCR. Cell proliferation was evaluated using cell counting kit-8 and flow cytometry. Cell migration and cell invasion were assessed by wound healing and transwell assay, respectively. The interactions between LINC00968 and miR-22-5p were validated by RNA immunoprecipitation, RNA pull down and luciferase reporter assay.

Results: We found that IncRNA LINC00968 was significantly down-regulated in LUAD tissues and cell lines. LINC00968 level was positively correlated to survival rate, and negatively correlated to tumor node metastasis stage, tumor size and lymph node metastasis of LUAD patients. LINC00968 over-expression in LUAD cells inhibited cell proliferation and induced cell cycle arrest at G1 phase. LINC00968 overexpression also suppressed migration, invasion and epithelial mesenchymal transition (EMT) as evidenced by elevated E-cadherin, decreased N-cadherin, TWIST and SNAIL levels. We further validated that LINC00968 localized in cytoplasma and acted as an upstream of microRNA miR-22-5p, which was up-regulated in LUAD tissues and cell lines. Besides, elevated miR-22-5p expression abolished the effect of LINC00968 over-expression on LUAD progression including in vivo tumor growth. In addition, we first validated that cell division cycle 14A (CDC14A), which was down-regulated in LUAD tissues, was a downstream target of miR-22-5p. We over-expressed CDC14A in LUAD cells and miR-22-5p induced LUAD progression was partially reversed.

Conclusion: our study demonstrated that LINC00968 inhibited proliferation, migration and invasion of LUAD by sponging miR-22-5p and further restoring CDC14A. This novel regulatory network might provide us with promising diagnostic and therapeutic target in LUAD treatment.

\section{Background}

Lung cancer remains one of the leading causes of cancer death in China and worldwide. Accounting for $21.9 \%$ of all cancer cases in 2018 , lung cancer is the most common diagnosed cancer in male in China, which is mainly attribute to tobacco intake and absence of efficient early-stage diagnosis and treatment strategies[1, 2]. Non-small-cell lung carcinoma (NSCLC) accounts for over $85 \%$ of all lung cancer cases, among which lung adenocarcinoma (LUAD) is the most common subtype [3]. LUAD is an aggressive human cancer which is resistant to conventional radiotherapy and chemotherapy. LUAD patients are often diagnosed at advanced stages, accompanying with disseminated metastasis and leaving them with limited treat options [4]. Smoking and exposure to radon, non-industrial air-pollution, asbestos and some chemicals are major risk factors for LUAD [5]. In spite of tremendous efforts we made in 
understanding the pathogenesis of LUAD in the past decades, the precise underlying mechanisms of LUAD progression continues to be enigmatic.

Long noncoding RNAs (IncRNAs) refer to non-protein coding RNA transcripts containing more than 200 nucleotides [6]. Although increasing evidences revealed that IncRNAs are involved in numerous human diseases, cancer included, full understanding of their exact molecular mechanisms is still far beyond completion [7]. Long intergenic non-protein coding RNA 968 (LINC00968) is reported to be dysregulated in several human cancers including breast cancer, osteosarcoma, and epithelial ovarian cancer [8-10]. Integrative microarray analysis showed that LINC00968 is significantly down-regulated in NSCLC tissues compared with normal lung tissues $[11,12]$. Yet the precise role that LINC00968 plays in LUAD progression remains unclear. MicroRNAs are another group of noncoding RNAs, approximately 22 nucleotides in length, which participate in a variety of physiological processes by inhibiting the expression of target genes [13]. MiR-22-5p was identified as a potential biomarker for acute myocardial infarction and gastric cardia adenocarcinoma [14-16]. Interestingly, Human MicroRNA Expression Database (HMED) shows that miR-22-5p is significantly up-regulated in lung cancer tissues. LINC00968 overexpression in human LUAD cell line A549 results in down-regulation of miR-22-5p level [17]. Besides, bioinformatics prediction on LncTar database (http://www.cuilab.cn/Inctar) demonstrated that LINC00968 could directly bind to miR-22-5p [18]. Consequently, we hypothesize that LINC00968 regulates LUAD progression through competitive binding to miR-22-5p. In addition, we found that cell division cycle 14A (CDC14A), a member of dual specificity protein tyrosine phosphatase family, was significantly downregulated in LUAD tissues (Gene Expression Profiling Interactive Analysis, GEPIA) [19]. CDC14A also acts as a putative down-stream target of miR-22-5p as predicted on TargetScan database (http://www.targetscan.org/vert_72/). CDC14A was involved in the regulation of cell cycle and DNA repair, suggesting its crucial role in cancer progression [20]. Yet whether miR-22-5p regulates LUAD progression by targeting CDC14A remain elusive.

In the present study, we analyzed the correlation between LINC00968 level and clinicopathological characteristics of LUAD patients. Then we examined the expression levels of LINC00968, miR-22-5p and CDC14A in LUAD tissues. We also investigated the effect of LINC00968, miR-22-5p and CDC14A on proliferation, migration and invasion of LUAD cells. Finally, we validated the predicted correlation.

\section{Materials And Methods}

\section{Clinical samples and ethics statement}

All LUAD tissues and adjacent non-tumor lung tissues $(n=60)$ were collected with the consent of patients. All experiment regarding clinical samples were performed in accordance with the ethical guidelines of the Declaration of Helsinki and approved by the Ethics Committee of International Medical Center Hospital.

\section{Cell culture and transfection}


Normal lung epithelial cell line BEAS2B, LUAD cell lines A549 and H1975 were purchased from Procell (China). LUAD cell lines HCC2935 and PC-9 were obtained from the American type culture collection (ATCC) cell bank. BEAS2B, A549 and H1975 cells were cultured with dulbecco's modified eagle medium (DMEM) containing 10\% fatal bovine serum (FBS). HCC2935 and PC-9 cells were maintained in Roswell Park Memorial Institute-1640 (RPMI-1640) medium with 10\% FBS. LINC00968 was inserted into lentiviral vector pRLenti-CMV-MCS-3FLAG (OBIO, China) for over-expression. LINC00968 lentivirus and negative control (NC) lentivirus, termed as LV-LINC00968 and LV-NC, were packed and amplified in HEK293T cells. MiR-22-5p mimics, non-specific (NC) mimics, miR-22-5p agomir, NC agomir and NC agomir were synthesized in GenePharma (China). CDC14A was inserted into pcDNA3.1 between Xho I and BamH I for over-expression, termed as pcDNA3.1-CDC14A. Cells were transfected with mimics, agomirs and pcDNA3.1 vectors using lipofectamine2000 according to the manufactures' instruction.

\section{Quantitative real-time PCR}

LUAD and adjacent non-tumor tissues were smashed using a grinder. Patient samples and cell lines were lysed with Trizol reagent (ThermoFisher, USA) to extract total RNAs, which was further reversely transcribed into cDNA using SuperScript IV reverse transcriptase (ThermoFisher, USA). Then cDNA products were quantified by real-time PCR using SYBR Green (Solarbio, China). Housekeeping gene GAPDH was used as an internal control for LINC00968 and CDC14A, RNU6B was used as an internal control for miR-22-5p. Results from quantitative real-time was analyzed using $g$ the $2^{-\triangle \triangle C T}$ method. All primers used were listed as below:

LINC00968-forward: 5'-CCACTCCTTTAGTCGTTGTGC-3'; LINC00968-reverse: 5'GGTCCCTCATTCCTATCCC-3';

MiR-22-5p-forward: 5'-AGTTCTTCAGTGGCAAGCTTTA-3'; miR-22-5p-reverse: 5'-TGGTGTCGTGGAGTCG-3';

CDC14A-forward: 5'-GGGGAACTAATCGGGGCTTG-3'; CDC14A-reverse: 5'-CAGCGGTCCAAAATCTGCATA-3'; GAPDH-forward: 5'-ACAACTTTGGTATCGTGGAAGG-3'; GAPDH-reverse: 5'-GCCATCACGCCACAGTTTC-3';

RNU6B-forward: 5'-CTCGCTTCGGCAGCACA-3; RNU6B-reverse: 5'-TGGTGTCGTGGAGTCG-3';

\section{Cell counting kit-8 (CCK-8)assay}

A549 and H1975 cells showed relative lower LINC00968 level among four LUAD cell lines, thus they were chosen for subsequent investigations. Cell proliferation was determined using cell counting kit-8 (MedChemExpress, USA) following the manufactures' instruction. A549 and $\mathrm{H} 1975$ cells were seeded into a 96 -well plate $\left(2 \times 10^{4}\right.$ cells/well $)$ and cultured for 24 hours. Cells were treated with LV-LINC00968/LVNC, miR-22-5p agomir/NC-agomir or pcDNA3.1-CDC14A/vector, followed by incubation for 24 hours, 48 hours, 72 hours and 96 hours. Then cells were incubated with $10 \mu \mathrm{l} \mathrm{CCK-8} \mathrm{solution} \mathrm{for} 3$ hours and absorbance at $450 \mathrm{~nm}$ was measured using a microplate reader.

\section{Cell cycle detection}


A549 and H1975 cells were harvested and re-suspended with PBS buffer after treatment. Cells were fixed with $1 \mathrm{ml}$ pre-cooled $70 \%$ ethanol for 2 hours and rinsed with PBS buffer. Then cells were incubated with $500 \mu$ propidium iodide $(50 \mu \mathrm{g} / \mathrm{ml})$ for $37^{\circ} \mathrm{C}$ for 30 minutes, followed by flow cytometry detection within 24 hours.

\section{Wound healing and transwell assay}

Wound healing assay was used to assess cell migration. A549 and H1975 cells were seeded into a 6-well plate and cultured to confluence, scratch on the cell momolayer was performed using a $200 \mu$ piptte tip, followed by observation under a microscope 24 hours after scratch. Transwell assay was performed to evaluate cell invasive capacity. A549 and H1975 cells were seeded into the Matrigel pre-coated upper chamber and cultured with serum-free culture medium for 48 hours, and lower chamber with culture medium containing 20\% FBS was used as an attractant. Cells invaded to the lower chamber were fixed with $4 \%$ paraformaldehyde and stained with $1 \%$ crystal violet for 5 minutes, followed by cell counting under a microscope.

\section{Western blotting}

A549 and H1975 cells were harvested and lysed with RIPA lysis buffer (Beyotime, China) after treatment, followed by quantification with commercial QuantiPro BCA assay kit (Sigma, USA). Then equivalent amount of total proteins were fractionated on SDS-polyacrylamide gel and then transferred onto a polyvinylidene fluoride (PVDF) membrane (ThermoFisher, USA). The PVDF membrane was sealed with $5 \%$ skim milk and incubated with primary antibodies overnight at $4{ }^{\circ} \mathrm{C}$. After rinsing with PBS buffer for three times, the PVDF membrane were incubated with secondary antibodies for 60 minutes at room temperature. Then PVDF membrane was illuminated with ECL luminescence reagent (Sangon, China), and relative protein levels were analyzed using IPP6.0. Primary antibodies used in this study were Ecadherin antibody (1:1000, SinoBiological, China), N-cadherin antibody (1:1000, SinoBiological, China), SNAIL antibody (1:1000, Affinity, USA), TWIST antibody (1:1000, Affinity, USA) and CDC14A antibody (1:1000, Affinity, USA). HRP- conjugated goat anti-rabbit antibody (1:5000, Affinity, USA) was used as secondary antibody.

\section{Luciferase reporter assay}

The interactions between LINC00968 and miR-22-5p and between miR-22-5p and CDC14A were validated using luciferase reporter assay. In brief, mutant type of LINC00968 and CDC14A, termed as LINC00968MUT and CDC14A-MUT, absent miR-22-5p binding site were obtained using overlapping PCR. Wildtype and mutant type of LINC00968 and CDC14A were amplified and cloned into pmiRGLO vector. The recombinant pmiRGLO plasmids were co-transfected with miR-22-5p/NC mimics into A549 and H1975 cells. The binding activity was assessed by measuring absorbance at $560 \mathrm{~nm}$ using commercial Luciferase Reporter Assay Kit (ThermoFisher, USA).

\section{Fluorescent in situ hybridation (FISH)}


FISH assay was performed to detect the subcellular localization of LINC00968 in LUAD cells. In brief, A549 and $\mathrm{H} 1975$ cells were baked at $37{ }^{\circ} \mathrm{C}$ for two hours and dehydrated in $50 \%, 80 \%$ and $98 \%$ gradient ethanol for 3 minutes. Then A549 and H1975 cells were hybridized with cy3-labelled LINC00968 (RiboBio, China) probe or NC probe (RiboBio, China) for 16 hours at $42{ }^{\circ} \mathrm{C}$. After staining with DAPI avoiding light for 5 minutes, typical images were captured under a microscope.

\section{RNA immunoprecipitation (RIP)}

The enrichment of LINC00968 and miR-22-5p on Argonaute 2 (Ago2) protein was evaluated using RIP assay kit (MBL life science, Japan). A549 and H1975 cells were lysed using $200 \mu$ RIP lysis buffer, cell lysate was centrifuged and $15 \mu \mathrm{l}$ of supernatant was used as input. The rest of supernatant protein was incubated with anti-Ago2-coated agarose beads (MBL life science, Japan) overnight at $4^{\circ} \mathrm{C}$, rabbit IgG was used as a control. The expression of Ago2 was detected by subsequent western blotting, and expression of LINC00968 and miR-22-5p was measured by quantitative real-time PCR.

\section{RNA pull down}

A549 and H1975 cells were lysed using lysis buffer containing RNase inhibitor and proteinase inhibitor. Cell lysate was centrifuged and re-suspended with hybridation buffer. Then cell lysate was incubated with biotinylated miR-22-5p or NC probes for 5 hours and incubated with magnetic streptavidin beads overnight at $4{ }^{\circ} \mathrm{C}$. The enriched RNA was purified and analyzed using quantitative real-time PCR.

\section{In vivo tumor growth}

Healthy 6 to 8-week old C57BL/6J mice were purchased from the experimental animal center of BIOCYTOGEN (China). A549 cells were cultured to exponential phase, infected with LV-LINC00968/LV-NC and miR-22-5p agomir. Then A549 cells $\left(2 \times 10^{6}\right.$ cells/mice) were subcutaneously injected to mice to establish tumor xenograft model $(n=6)$. Tumor volumes were measured every week for five weeks, and mice were euthanized at fifth week. Tumor weight was recorded and tumor tissues were fixed with $4 \%$ paraformaldehyde for subsequent detection. All animal experiments were performed following the guidelines for the care and use of laboratory animals and approved by the Ethics Committee of International Medical Center Hospital.

\section{Immunohistochemistry}

Immunohistochemistry assay was performed to detect the expression of Ki67 in mouse tumor tissues. In brief, $5 \mu \mathrm{m}$ of tissue sections were deparaffinized and boiled with $10 \mathrm{mM}, \mathrm{pH} 6.0$ citric acid buffer for 15 minutes for antigen retrieval. Then tissue sections were blocked with $5 \%$ goat serum for one hour and incubated with Ki67 antibody (1:200, SinoBiological, China) overnight at $4^{\circ} \mathrm{C}$. After washing with TBS buffer ( $0.1 \%$ Tween 20 in PBS buffer), tissue sections were incubated with HRP-conjugated secondary antibody and Ki67 signal was developed using 3, 3'-diaminobenzidine ((Sangon, China)).

\section{Statistical analysis}


All results in our study were presented as means \pm SD and all data analysis was conducted using Graphpad Prism 8.0. Mean values between two groups were analyzed by Students' t-test. Survival rate was analyzed using Log-rank (Mantel-Cox) test. Data from Cell counting kit-8 assay, tumor growth and luciferase reporter assay were analyzed by Two-way ANOVA. The correlation between LINC00968 level and clinicpathological characteristics of LUAD patients were analyzed using Pearson $\chi 2$ test. The rest of results were analyzed using One-way ANOVA. All experiments were repeated for more than three times and $p$ values less than 0.05 were considered as statistically significant.

\section{Results}

\section{LINC00968 was significantly down-regulated in LUAD tissues and cell lines}

TCGA database showed that LINC00968 was significantly down-regulated in LUAD tissues (Fig. 1A). We first examined the LINC00968 level in 60 LUAD patients. LUAD tissues showed significantly lower LINC00968 level compared with adjacent non-tumor tissues (Fig. 1B). Further follow-up visit showed that LUAD patients with relative high LINC00968 level have significantly higher survival rate in contrast with that with relative low LINC00968 level (Fig. 1C). Then we analyzed the correlation between LINC00968 level and clinicpathological characteristics of LUAD patients. Tumor node metastasis (TNM) stage, tumor size and Lymph node metastasis were negatively correlated to LINC00968 level (Table.1). Besides, we detected the LINC00968 level in normal lung epithelial cell line BEAS2B and four LUAD cell lines, LUAD cells showed significantly lower LINC00968 level compared with BEAS2B cells, especially in A549 and H1975 cells (Fig. 1D). These results implied that LINC00968 may serve as a crucial regulator of LUAD progression.

\section{LINC00968 over-expression inhibited proliferation, migration and invasion of LUAD cells}

We over-expressed LINC00968 in A549 and H1975 cells using lentivirus vector, and LINC00968 level was elevated for more than twice in LV-LINC00968 group (Fig. 2A). We detected cell proliferation using CCK-8 assay, LV-LINC00968 significantly inhibited proliferation in both A549 and H1975 cells 48 hours after infection compared with LV-NC (Fig. 2B). Cell cycle detection by flow cytometry showed that cells at G1 phase were increased, while cells at S phase and G2 phase were reduced in both A549 and H1975 cells after LV-LINC00968 infection (Fig. 2C). Wound healing assay revealed that LINC00968 over-expression remarkably suppressed the migratory ability of in A549 and H1975 cells (Fig. 2D). Further transwell assay showed similar results, the number of LUAD cells invaded to lower chamber in LV-LINC00968 group was conspicuously fewer than that in LV-NC group (Fig. 2E). Besides, LINC00968 over-expression increased the E-cadherin protein level, while reduced the expression of N-cadherin, TWIST and SNAIL in both A549 and $\mathrm{H} 1975$ cells (Fig. 2F). These results suggested that LINC00968 over-expression inhibited the malignance of LUAD cells. 


\section{LINC00968 was a putative regulator of miR-22-5p}

The mRNA level of miR-22-5p was significantly higher in LUAD patients compared with that in Normal control (Fig. 3A). Bioinformatics prediction indicated that LINC00968 might competitively bind to miR-225p (Fig. 3B), which inspired us to explore the interaction between LINC00968 and miR-22-5p. Further luciferase reporter assay showed that miR-22-5p mimics significantly inhibited the luciferase activity in LINC00968-WT group compared with NC mimics, whereas no obvious effect of miR-22-5p mimics on luciferase activity in LINC00968-MUT group was detected (Fig. 3C). Moreover, LV-LINC00968 infection significantly reduced the miR-22-5p level compared to LV-NC in both A549 and H1975 cells (Fig. 3D). FISH assay was performed to determine the subcellular localization of LINC00968, and LINC00968 was mainly localized in cytoplasm (Fig. 3E). RIP assay showed that Ago2 was successfully precipitated, accompanied with enrichment of LINC00968 and miR-22-5p in Ago2 complex (Fig. 3F). Besides, biotinylated miR-22-5p probe successfully pulled down LINC00968 compared with NC probe in both A549 and H1975 cells (Fig. 3G). These results revealed that LINC00968 acted as a putative regulator of miR-22$5 p$.

\section{LINC00968 inhibited proliferation, migration and invasion of LUAD cells through regulating miR-22-5p}

Next we investigated whether LINC00968 regulated the malignance of LUAD cells through regulating miR22-5p. A549 and H1975 cells infected with LV-LINC00968/LV-NC were transfected with miR-22-5p/NC agomir. LINC00968 over-expression significantly inhibited cell proliferation, while further miR-22-5p agomir transfection abolished the effect (Fig. 4A). Cells at G1 phase was increased whereas cells at $S$ phase was reduced after LV-LINC00968 infection, all these effect was partially reversed by miR-22-5p agomir transfection (Fig. 4B). Wound healing assay showed that LINC00968 over-expression inhibited cell migration while miR-22-5p agomir abrogated the effect in both A549 and H1975 cells (Fig. 4C). Similarly, LINC00968 over-expression reduced cell invasion number whereas further miR-22-5p agomir transfection abolished the effect (Fig. 4D). LINC00968 over-expression elevated the E-cadherin level, while reduced the protein levels of N-cadherin, TWIST and SNAIL, all these effects were partially reversed by further miR-22-5p agomir transfection (Fig. 4E). Besides, mice in LV-LINC00968 + NC agomir group showed significant smaller tumor size than that in LV-NC + NC agomir group, yet the tumor suppressive effect was partially reversed in LV-LINC00968 + miR-22-5p agomir group (Fig. 4F). We further examined the expression of Ki67 in mouse tumor tissues, LINC00968 obviously decreased the Ki67 protein level in vivo, whereas further miR-22-5p agomir treatment partially restored Ki67 expression (Fig. 4G). Taken these together, our results showed that LINC00968 inhibited proliferation, migration and invasion of LUAD cells via targeting miR-22-5p.

\section{MiR-22-5p promoted proliferation, migration and invasion of LUAD cells through targeting CDC14A}


In the last part, we investigated whether miR-22-5p regulated LUAD progression through targeting CDC14A. Quantitative real-time PCR assay showed that CDC14A was significantly down-regulated in LUAD tissues compared with adjacent non-tumor tissues (Fig. 5A). Luciferase reporter assay showed that miR-22-5p mimics notably inhibited the luciferase activity in wildtype CDC14A (CDC14A-WT) group, yet had no obvious impact on CDC14A-MUT (Fig. 5B). We transfected A549 and H1975 cells with miR-22-5p agomir, and significantly reduced mRNA level of CDC14A was detected (Fig. 5C). We further transfected cells with miR-22-5p agomir and pcDNA3.1-CDC14A. MiR-22-5p agomir significantly promoted the proliferation of LUAD cells, while this effect was reversed by simultaneous pcDNA3.1-CDC14A transfection (Fig. 5D). Similarly, flow cytometry detection revealed that miR-22-5p agomir significantly increased the number of cells at S phase yet reduced the G1 phase cells, whereas further CDC14A overexpression abrogated the effect (Fig. 5E). Besides, wound healing and transwell assay showed that miR22-5p agomir significantly facilitated the migration and invasion of LUAD cells, which was partially abolished by CDC14A over-expression (Fig. 5F, G). In addition, miR-22-5p agomir inhibited the expression of E-cadherin yet elevated the expression of N-cadherin, SNAIL and TWIST, all these effects were abrogated by further CDC14A over-expression (Fig. $5 \mathrm{H}$ ). These results suggested that miR-22-5p promoted the proliferation, migration and invasion of LUAD cells via targeting CDC14A.

\section{Discussion}

Large-scale cancer genomics projects revealed that numerous IncRNAs are aberrantly expressed in a variety of human cancers [21]. LncRNAs exert their diverse function in the tumorgenesis and tumor development via acting as oncogenes, tumor suppressors or regulatory RNAs [22]. However, to date only about $1 \%$ of all 3,000 human IncRNAs has been characterized [7]. LINC00968 was first identified by Hui et al in 2015, and the following researches demonstrated its diverse role in different human cancers. LINC00968 inhibited the progression of breast cancer yet acted as an oncogene in epithelial ovarian cancer and osteosarcoma [8-10,12]. Here in our study, we investigated the exact role of LINC00968 in the progression of LUAD. Tissue-specific expression analysis showed that LINC00968 was specifically expressed in lung [23]. We first detected the expression level of LINC00968 in LUAD tissues, and the down-regulation of LINC00968 was highly in accordance with previous researches [12, 24]. Lower LINC00968 level accompanied with lower survival rate, more advanced TNM stage, larger tumor size as well as more positive lymphnode metatstasis. All these results implied that LINC00968 are involved in LUAD progression and may serve as a novel diagnostic target. Then we cloned LINC00968 into lentivirus vector for over-expression in two LUAD cell lines which displayed lowest LINC00968 level. Uncontrolled malignant proliferation and cell cycle are critical characteristics of human cancers [25]. LINC00968 overexpression significantly inhibited the proliferation of LUAD cells and induced cell cycle arrest at G1 phase, further tumor xenograft assay showed that LINC00968 inhibited tumor growth in vivo, indicating that LINC00968 is a putative LUAD suppressor. Distant metastasis is responsible for majority of cancer death. Migration, invasion and epithelial mesenchymal transition (EMT) are most crucial procedures involving in tumor metastasis, which confer cancer cells with enhanced resistance to apoptotic stimuli [26]. Here we found that LINC00968 over-expression inhibited the migration and invasion of LUAD cells. EMT was also 
suppressed as evidenced by elevated E-cadherin and reduced EMT markers N-cadherin, SNAIL and TWIST [27]. Our findings demonstrated that LINC00968 over-expression inhibited LUAD progression and LINC00968 is a promising diagnostic and therapeutic target for LUAD treatment.

The "competing endogenous RNA" (ceRNA) hypothesized that IncRNAs could competitively bind to microRNAs and forms a new regulatory network across the transcriptome [28]. To date, the role of miR$22-5 p$ in LUAD progression is poorly understood. In the present study, we first found that miR-22-5p was significantly up-regulated in LUAD tissues and cell lines, indicating that miR-22-5p might serve as a potential early-stage biomarker for LUAD diagnosis. We also validated the interaction between LINC00968 and miR-22-5p in our study, which was highly consistent with previous miRNA microarray analysis [17]. Besides, LINC00968 over-expression-induced inhibited proliferation, migration and invasion of LUAD cells were significantly reversed by miR-22-5p supplement, indicating that LINC00968 may function as LUAD suppressor through sponging miR-22-5p and this novel regulatory network is a potential therapeutic target for LUAD management.

Cell cycle progression is mainly promoted by cyclins and cyclin-dependent kinases (CDKs), which makes them promising targets for cell cycle control and hence cancer therapy [29]. CDC14 phosphatase family is highly conserved among yeast and human. Active CDC14A reverses CDK phosphorylation and further regulates the mitotic entry via regulating $C D C 25 A$ and $C D C 25 B[30,31]$. Paulsen et al validated that CDC14A interacts with the CDK1/cyclin B complex, suggesting that CDC14A inhibits the activity of CDK1/cyclin B complex during interphase and therefore induces cell cycle arrest. Besides, CDC14A overexpression may activate p53 signaling and further induce cell apoptosis or cell cycle arrest [20]. In the present study, CDC14A was significantly down-regulated in both LUAD tissues and cell lines, which was in line with previous study [20,32]. In addition, we first validated that CDC14A was a downstream target of miR-22-5p, and LUAD progression facilitated by miR-22-5p was reversed by CDC14A over-expression, indicating that $\mathrm{CDC14A}$ was a putative tumor suppressor [32].

\section{Conclusion}

In conclusion, we validated a novel regulatory network in LUAD progression: LINC00968 inhibits proliferation, migration and invasion of LUAD through sponging miR-22-5p and further restoring CDC14A. LINC00968 and CDC14A were putative tumor suppressors and the novel regulatory network may offer us new therapeutic targets and strategies in LUAD management.

\section{List Of Abbreviations}

LINC00968: long intergenic non-protein coding RNA 968

CDC14A: cell division cycle 14A

LUAD: lung adenocarcinoma 
IncRNA: long noncoding RNA

LV-LINC00968: LINC00968 lentivirus

LC-NC: negative control lentivirus

FISH: fluorescent in situ hybridation

RIP: RNA immunoprecipitation

\section{Declarations}

\section{Ethics approval and consent to participate}

All experiment regarding clinical samples were performed in accordance with the ethical guidelines of the Declaration of Helsinki and approved by the Ethics Committee of International Medical Center Hospital. Clinical samples were collected with the consent of participants.

\section{Consent for publication}

Consent for publication was obtained from all participants.

\section{Availability of data and material}

Not applicable.

\section{Competing interests}

None.

\section{Funding}

None.

\section{Authors' contributions}

Chao Wu and Xuzhao Bian conceived and designed the study, and wrote the manuscript. Xinpeng Han provided clinical samples and relative data. Liyuan Zhang and Yang Wu performed the experiments. Tianli Pei reviewed the manuscript. All authors read and approved the manuscript.

\section{Acknowledgements}

Not applicable.

\section{References}


1. Fitzmaurice C, Abate D, Abbasi N, Abbastabar H, Abd-Allah F, Abdel-Rahman O, Abdelalim A, Abdoli A, Abdollahpour I, Abdulle ASM et al. Global, Regional, and National Cancer Incidence, Mortality, Years of Life Lost, Years Lived With Disability, and Disability-Adjusted Life-Years for 29 Cancer Groups, 1990 to 2017: A Systematic Analysis for the Global Burden of Disease Study. JAMA Oncol. 2019.

2. Feng RM, Zong YN, Cao SM, Xu RH. Current cancer situation in China: good or bad news from the 2018 Global Cancer Statistics? Cancer Commun (Lond). 2019; 39(1):22.

3. Zappa C, Mousa SA. Non-small cell lung cancer: current treatment and future advances. Transl Lung Cancer Res. 2016; 5(3):288-300.

4. Denisenko TV, Budkevich IN, Zhivotovsky B. Cell death-based treatment of lung adenocarcinoma. Cell Death Dis. 2018; 9(2):117.

5. Molina JR, Yang P, Cassivi SD, Schild SE, Adjei AA. Non-small cell lung cancer: epidemiology, risk factors, treatment, and survivorship. Mayo Clin Proc. 2008; 83(5):584-594.

6. Birney E, Stamatoyannopoulos JA, Dutta A, Guigo R, Gingeras TR, Margulies EH, Weng Z, Snyder M, Dermitzakis ET, Thurman RE et al. Identification and analysis of functional elements in $1 \%$ of the human genome by the ENCODE pilot project. Nature. 2007; 447(7146):799-816.

7. Tsai MC, Spitale RC, Chang HY. Long intergenic noncoding RNAs: new links in cancer progression. Cancer Res. 2011; 71(1):3-7.

8. Sun X, Huang T, Zhang C, Zhang S, Wang Y, Zhang Q, Liu Z. Long non-coding RNA LINC00968 reduces cell proliferation and migration and angiogenesis in breast cancer through up-regulation of PROX1 by reducing hsa-miR-423-5p. Cell Cycle. 2019; 18(16):1908-1924.

9. Liu G, Yuan D, Sun P, Liu W, Wu PF, Liu H, Yu GY. LINC00968 functions as an oncogene in osteosarcoma by activating the PI3K/AKT/mTOR signaling. J Cell Physiol. 2018; 233(11):86398647.

10. Yao N, Sun JQ, Yu L, Ma L, Guo BQ. LINC00968 accelerates the progression of epithelial ovarian cancer via mediating the cell cycle progression. Eur Rev Med Pharmacol Sci. 2019; 23(11):46424649.

11. Qiao F, Li N, Li W. Integrative Bioinformatics Analysis Reveals Potential Long Non-Coding RNA Biomarkers and Analysis of Function in Non-Smoking Females with Lung Cancer. Med Sci Monit. 2018; 24:5771-5778.

12. Yu H, Xu Q, Liu F, Ye X, Wang J, Meng X. Identification and validation of long noncoding RNA biomarkers in human non-small-cell lung carcinomas. J Thorac Oncol. 2015; 10(4):645-654.

13. Wilson RC, Doudna JA. Molecular mechanisms of RNA interference. Annu Rev Biophys. 2013; 42:217-239.

14. Wang Y, Chang W, Zhang Y, Zhang L, Ding H, Qi H, Xue S, Yu H, Hu L, Liu D et al. Circulating miR-22$5 p$ and miR-122-5p are promising novel biomarkers for diagnosis of acute myocardial infarction. $J$ Cell Physiol. 2019; 234(4):4778-4786.

15. Li H, Zhang P, Li F, Yuan G, Wang X, Zhang A. Plasma miR-22-5p, miR-132-5p, and miR-150-3p Are Associated with Acute Myocardial Infarction. Biomed Res Int. 2019; 2019:5012648. 
16. Wang J, Zhang H, Zhou X, Wang T, Zhang J, Zhu W, Zhu H, Cheng W. Five serum-based miRNAs were identified as potential diagnostic biomarkers in gastric cardia adenocarcinoma. Cancer Biomark. 2018; 23(2):193-203.

17. Li DY, Chen WJ, Luo L, Wang YK, Shang J, Zhang Y, Chen G, Li SK. Prospective IncRNA-miRNA-mRNA regulatory network of long non-coding RNA LINC00968 in non-small cell lung cancer A549 cells: A miRNA microarray and bioinformatics investigation. Int J Mol Med. 2017; 40(6):1895-1906.

18. Li J, Ma W, Zeng P, Wang J, Geng B, Yang J, Cui Q. LncTar: a tool for predicting the RNA targets of long noncoding RNAs. Brief Bioinform. 2015; 16(5):806-812.

19. Tang Z, Li C, Kang B, Gao G, Zhang Z. GEPIA: a web server for cancer and normal gene expression profiling and interactive analyses. Nucleic Acids Res. 2017; 45(W1):W98-W102.

20. Paulsen MT, Starks AM, Derheimer FA, Hanasoge S, Li L, Dixon JE, Ljungman M. The p53-targeting human phosphatase hCdc14A interacts with the Cdk1/cyclin B complex and is differentially expressed in human cancers. Mol Cancer. 2006; 5:25.

21. Maurano MT, Humbert R, Rynes E, Thurman RE, Haugen E, Wang H, Reynolds AP, Sandstrom R, Qu H, Brody $\mathrm{J}$ et al. Systematic localization of common disease-associated variation in regulatory DNA. Science. 2012; 337(6099):1190-1195.

22. Arun G, Diermeier SD, Spector DL. Therapeutic Targeting of Long Non-Coding RNAs in Cancer. Trends Mol Med. 2018; 24(3):257-277.

23. Fagerberg L, Hallstrom BM, Oksvold P, Kampf C, Djureinovic D, Odeberg J, Habuka M, Tahmasebpoor $\mathrm{S}$, Danielsson A, Edlund $\mathrm{K}$ et al. Analysis of the human tissue-specific expression by genome-wide integration of transcriptomics and antibody-based proteomics. Mol Cell Proteomics. 2014; 13(2):397406.

24. Acha-Sagredo A, Uko B, Pantazi P, Bediaga NG, Moschandrea C, Rainbow L, Marcus MW, Davies MPA, Field JK, Liloglou T. Long non-coding RNA dysregulation is a frequent event in non-small cell lung carcinoma pathogenesis. Br J Cancer. 2020.

25. Feitelson MA, Arzumanyan A, Kulathinal RJ, Blain SW, Holcombe RF, Mahajna J, Marino M, MartinezChantar ML, Nawroth R, Sanchez-Garcia I et al. Sustained proliferation in cancer: Mechanisms and novel therapeutic targets. Semin Cancer Biol. 2015; 35 Suppl:S25-S54.

26. Mittal V. Epithelial Mesenchymal Transition in Tumor Metastasis. Annu Rev Pathol. 2018; 13:395412.

27. Fazilaty H, Rago L, Kass Youssef K, Ocana OH, Garcia-Asencio F, Arcas A, Galceran J, Nieto MA. A gene regulatory network to control EMT programs in development and disease. Nat Commun. 2019; 10(1):5115.

28. Salmena L, Poliseno L, Tay Y, Kats L, Pandolfi PP. A ceRNA hypothesis: the Rosetta Stone of a hidden RNA language? Cell. 2011; 146(3):353-358.

29. Otto T, Sicinski P. Cell cycle proteins as promising targets in cancer therapy. Nat Rev Cancer. 2017; $17(2): 93-115$. 
30. Vazquez-Novelle MD, Esteban V, Bueno A, Sacristan MP. Functional homology among human and fission yeast Cdc14 phosphatases. J Biol Chem. 2005; 280(32):29144-29150.

31. Vazquez-Novelle MD, Mailand N, Ovejero S, Bueno A, Sacristan MP. Human Cdc14A phosphatase modulates the G2/M transition through Cdc25A and Cdc25B. J Biol Chem. 2010; 285(52):4054440553.

32. Li L, Ernsting BR, Wishart MJ, Lohse DL, Dixon JE. A family of putative tumor suppressors is structurally and functionally conserved in humans and yeast. J Biol Chem. 1997; 272(47):2940329406.

\section{Table}

Table 1

\begin{tabular}{|c|c|c|c|c|c|}
\hline \multirow[t]{2}{*}{ Variable } & \multirow[t]{2}{*}{$\mathbf{n}$} & \multicolumn{2}{|c|}{ Relative LINC00968 level } & \multirow[t]{2}{*}{$c^{2}$} & \multirow[t]{2}{*}{$p$ value } \\
\hline & & High & Low & & \\
\hline Age (years) & & & & 0.271 & 0.794 \\
\hline$>65$ & 26 & 12 & 14 & & \\
\hline$£ 65$ & 34 & 18 & 16 & & \\
\hline Gender & & & & 0.537 & 0.642 \\
\hline Male & 38 & 17 & 21 & & \\
\hline Female & 22 & 12 & 10 & & \\
\hline TNM stage & & & & 5.384 & 0.039 \\
\hline I-II & 24 & 16 & 8 & & \\
\hline III-IV & 36 & 13 & 23 & & \\
\hline Tumor size & & & & 9.160 & 0.006 \\
\hline$£ 5 \mathrm{~cm}$ & 25 & 17 & 8 & & \\
\hline$>5 \mathrm{~cm}$ & 35 & 10 & 25 & & \\
\hline Lymph node metastasis & & & & 5.831 & 0.032 \\
\hline Negative & 22 & 14 & 8 & & \\
\hline Positive & 38 & 12 & 26 & & \\
\hline Smoking history & & & & 0.012 & 0.871 \\
\hline Smokers & 39 & 21 & 18 & & \\
\hline Non-smokers & 21 & 11 & 10 & & \\
\hline
\end{tabular}

The correlation between LINC00968 RNA level and clinicopathological characteristics of lung adenocarcinoma patients was analyzed using chi-square tests.

\section{Figures}


A

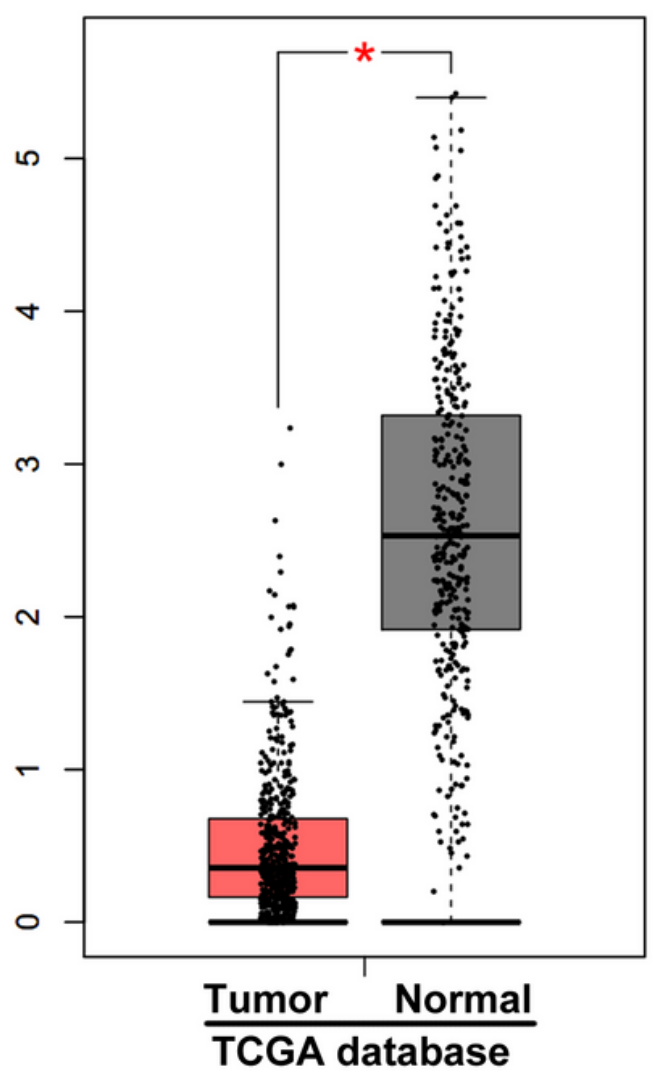

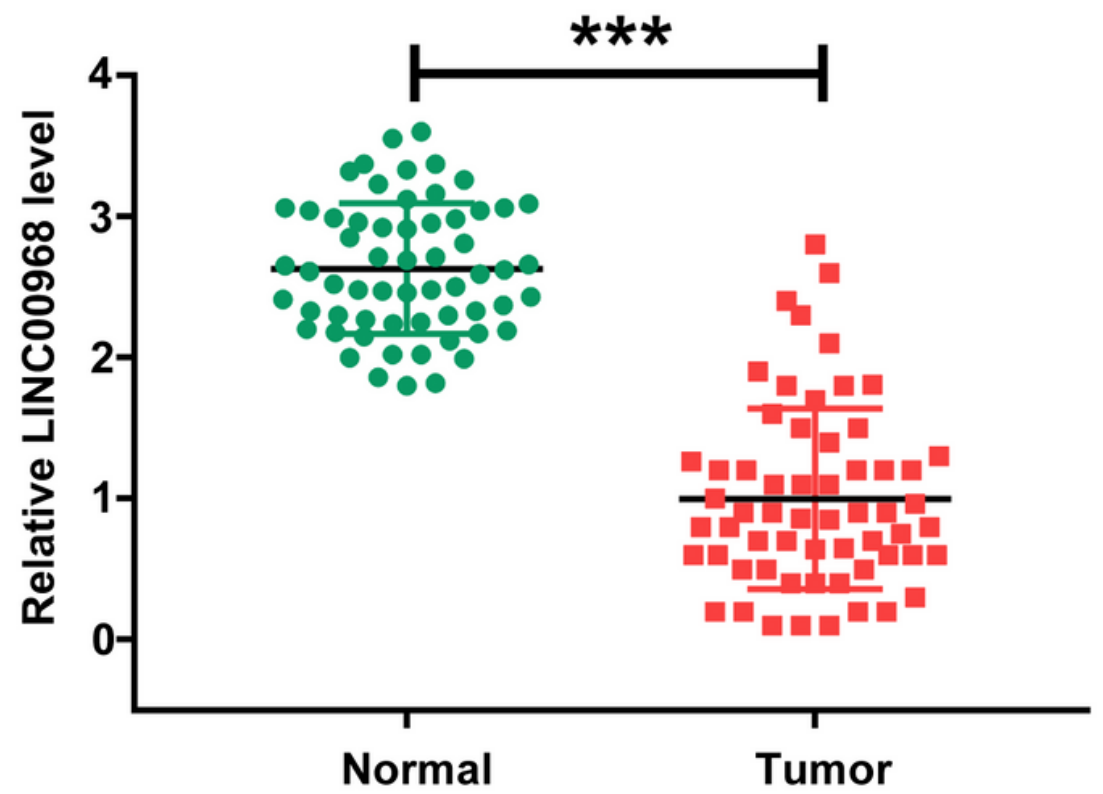

C

D
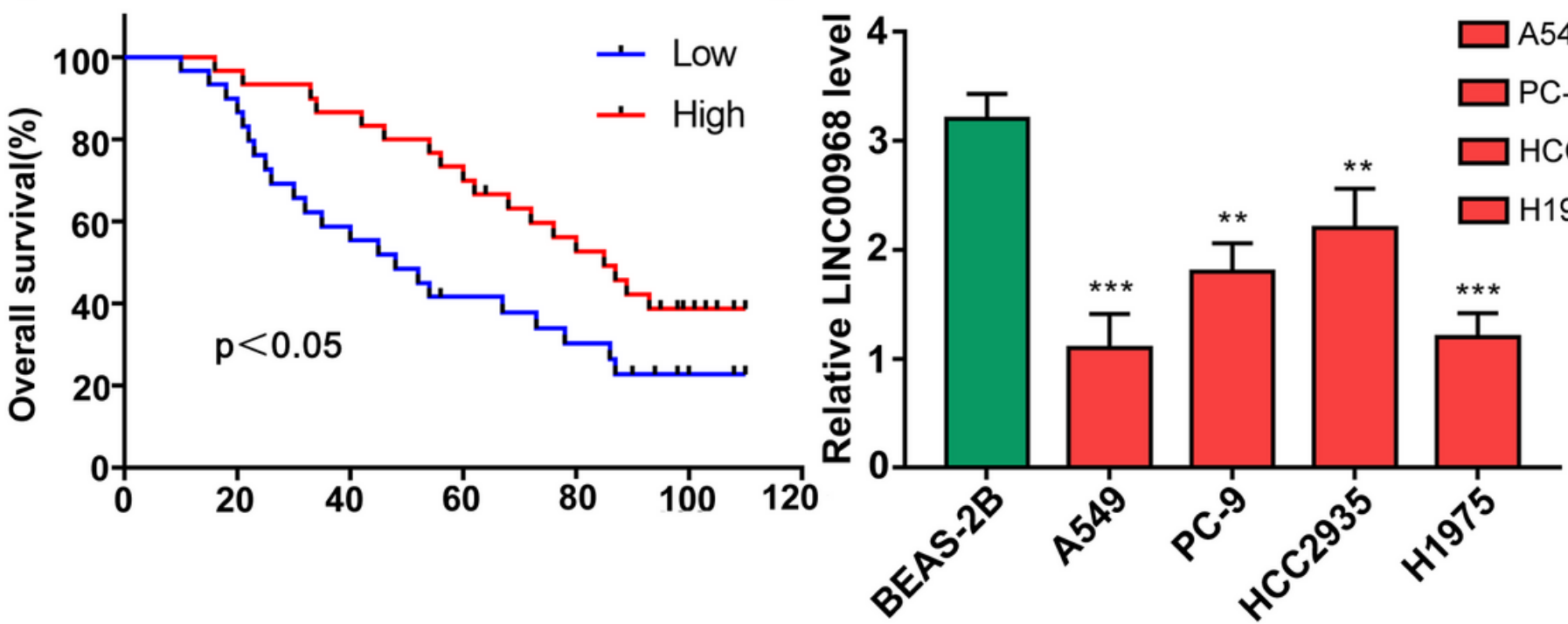

Figure 1

LINC00968 was down-regulated in LUAD tissues and cell lines. (A) The expression of LINC00968 in LUAD tissues and Normal tissues in TCGA database. (B) The LINC00968 level in LUAD tissues and adjacent non-tumor tissues in 60 LUAD patients was measured using quantitative real-time PCR. (C) Analysis of correlation between LINC00968 level and overall survival rate and time of LUAD patients. (D) The relative LINC00968 level in normal lung epithelial cell line BEAS2B and four LUAD cell lines was detected using quantitative real-time PCR. LINC00968: long intergenic non-protein coding RNA 968; LUAD: lung adenocarcinoma; ${ }^{*} p<0.05,{ }^{* *} p<0.01,{ }^{* \star *} p<0.001$. 

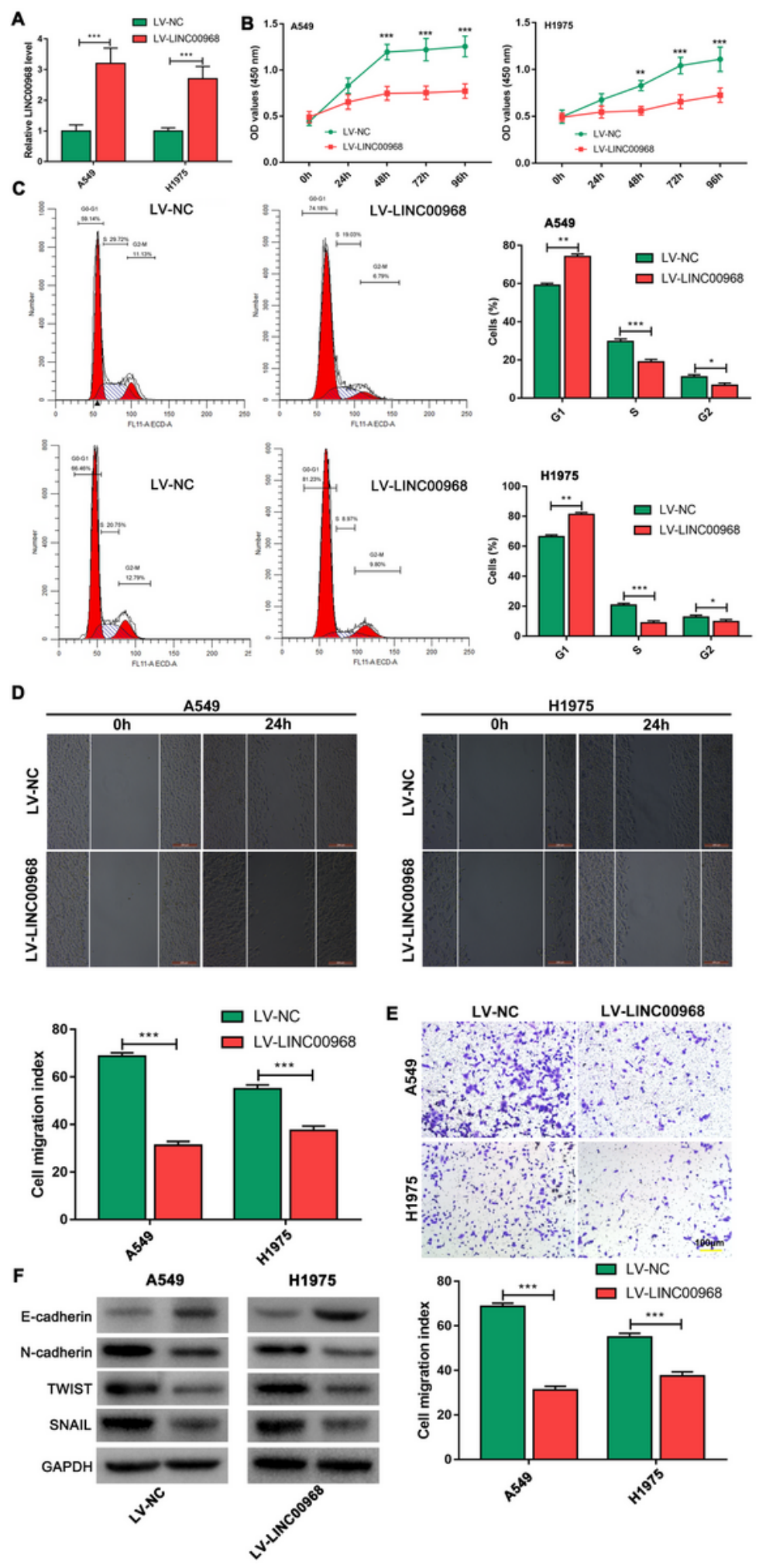

\section{Figure 2}

LINC00968 over-expression inhibited proliferation, migration and invasion of LUAD cells. A549 and H1975 cells were infected with LV-VC and LV-LINC00968. (A) Quantitative real-time PCR was performed to measure LINC00968 level in A549 and H1975 cells. (B) Cell counting kit-8 assay was carried out to evaluate cell proliferation. (C) Cell cycle of A549 and H1975 cells was detected using flow cytometry. (D) Wound healing assay was performed to assess the cell migration ability. (E) Cell invasion capacity was 
assessed using transwell assay. (F) The protein levels of E-cadherin, N-cadherin, TWIST and SNAIL was determined using western blotting. LINC00968: long intergenic non-protein coding RNA 968; LUAD: lung adenocarcinoma; LV-NC: negative control lentivirus; LV-LINC00968: LINC00968 over-expression lentivirus; ${ }^{*} \mathrm{p}<0.05,{ }^{*} \mathrm{p}<0.01, * * * \mathrm{p}<0.001$.

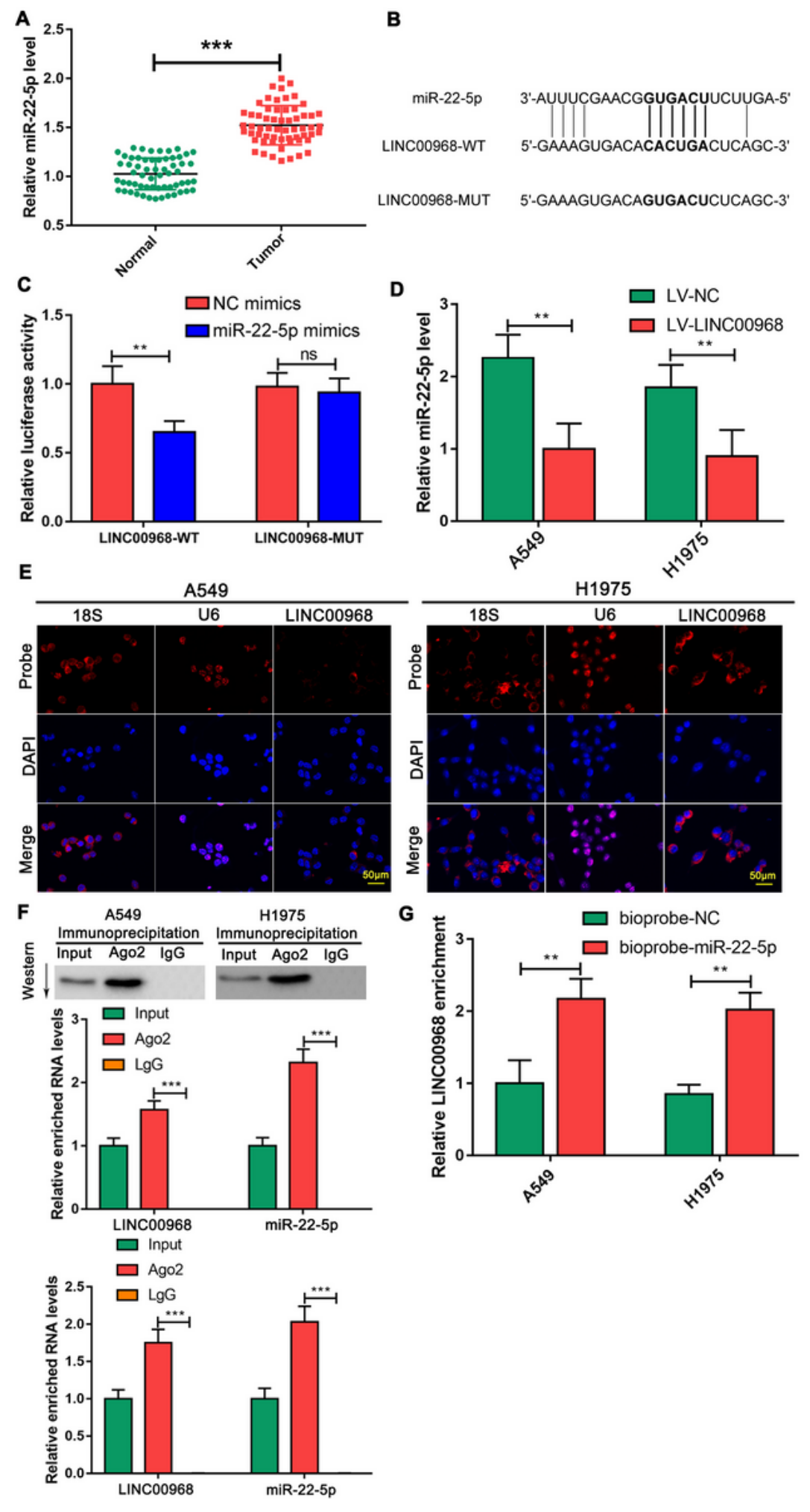

Figure 3 
LINC00968 was a putative regulator of miR-22-5p. (A) The relative miR-22-5p level in LUAD tissues and adjacent non-tumor tissues was measured using quantitative real-time PCR. (B) The binding site of miR22-5p on LINC00968 was shown in Fig. 3B. (C) The interaction between LINC00968 and miR-22-5p was validated by luciferase reporter assay. (D) The relative miR-22-5p level in A549 and H1975 cells after LINC00968 over-expression was measured using quantitative real-time PCR. (E) The subcellular localization of LINC00968 was displayed using FISH assay. (F) The enrichment of LINC00968 and miR22-5p on Ago2 complex was determined using RNA immunoprecipitation assay. (G) The enrichment of LINC00968 using biotinylated miR-22-5p probe was detected using quantitative real-time PCR.LINC00968: long intergenic non-protein coding RNA 968; LUAD: lung adenocarcinoma; FISH: fluorescent in situ hybridation; ${ }^{* *} p<0.01,{ }^{\star \star *} p<0.001$, ns: no significant. 

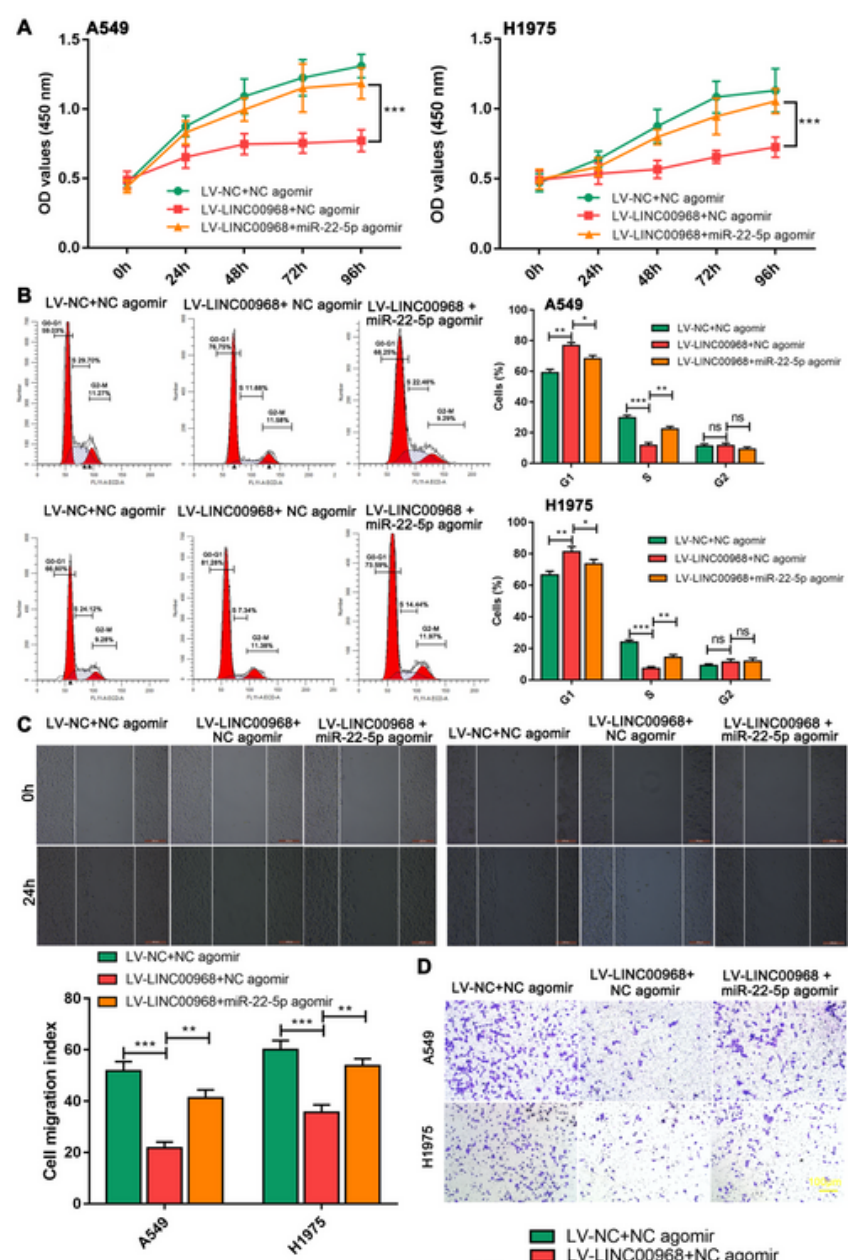

D LV-NC+NC agomir LV-LINCO0968+ LV-LINC00968 +
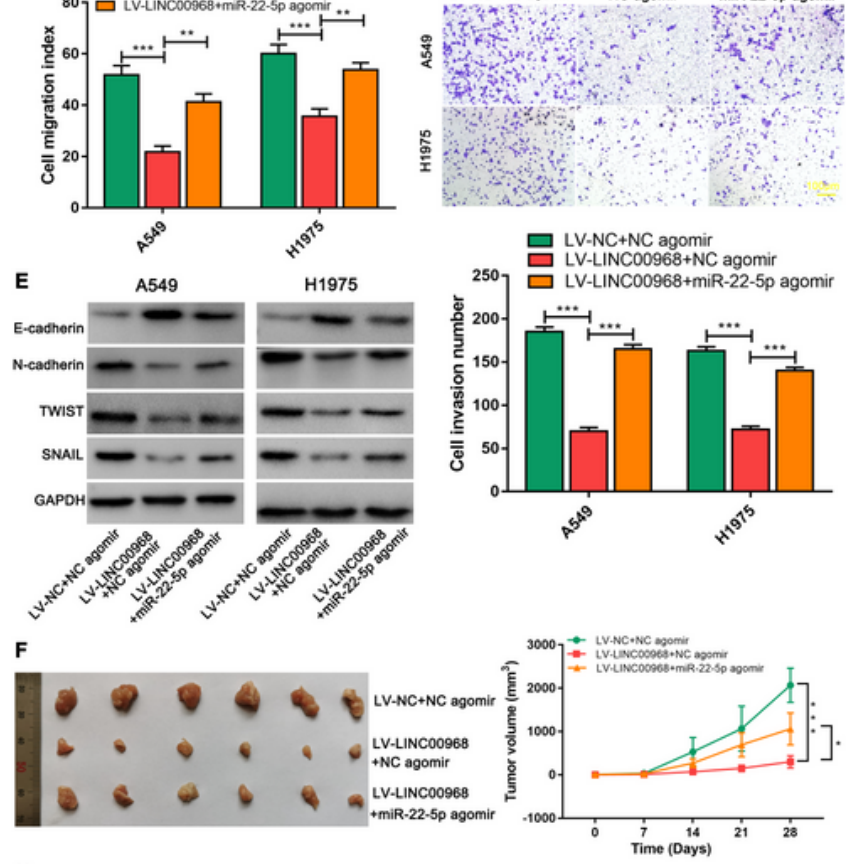

G

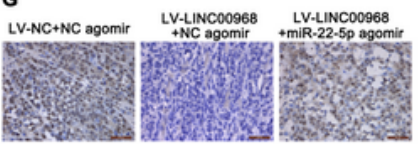

Figure 4

LINC00968 inhibited proliferation, migration and invasion of LUAD cells through regulating miR-22-5p. A549 and H1975 were infected with LV-LINC00968/LV-NC and transfected with miR-22-5p/NC agomir. (A) Cell proliferation was evaluated using cell counting kit-8 assay. (B) Cell cycle was detected using flow cytometry. (C) Wound healing assay was performed to assess cell migration. (D) Transwell assay was carried out to assess cell invasion. (E) The protein levels of E-cadherin, N-cadherin, TWIST and SNAIL was 
determined using western blotting. (F) A549 cells were subcutaneously injected to mice to form tumor xenograft model, and tumor volumes were analyzed. (G) The expression of Ki67 in mouse tumor tissues was determined by immunohistochemistry. LINC00968: long intergenic non-protein coding RNA 968; LUAD: lung adenocarcinoma; LV-NC: negative control lentivirus; LV-LINC00968: LINC00968 overexpression lentivirus; ${ }^{*} \mathrm{p}<0.05,{ }^{* *} \mathrm{p}<0.01,{ }^{* * *} \mathrm{p}<0.001$.
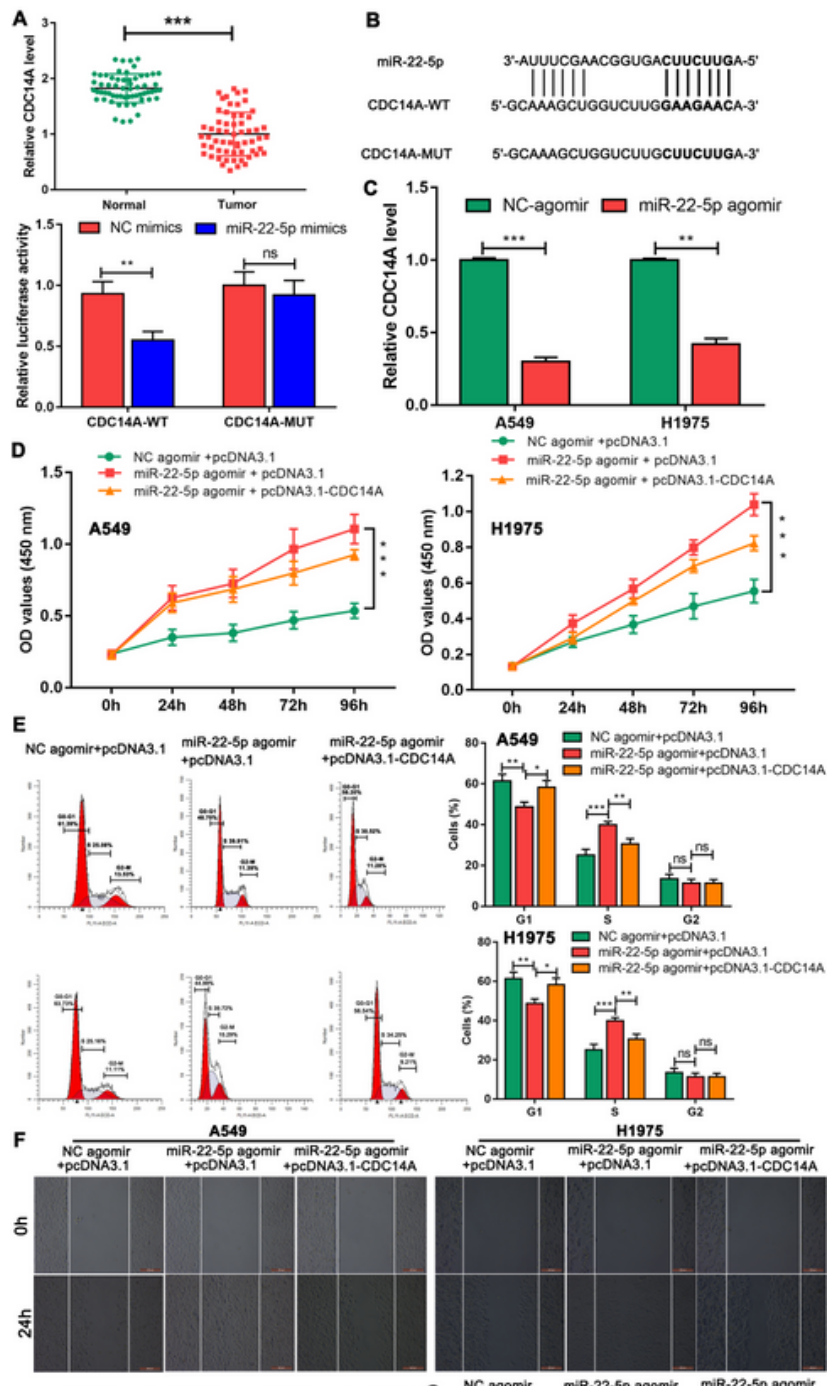

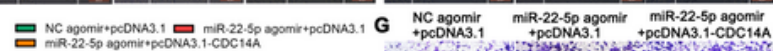
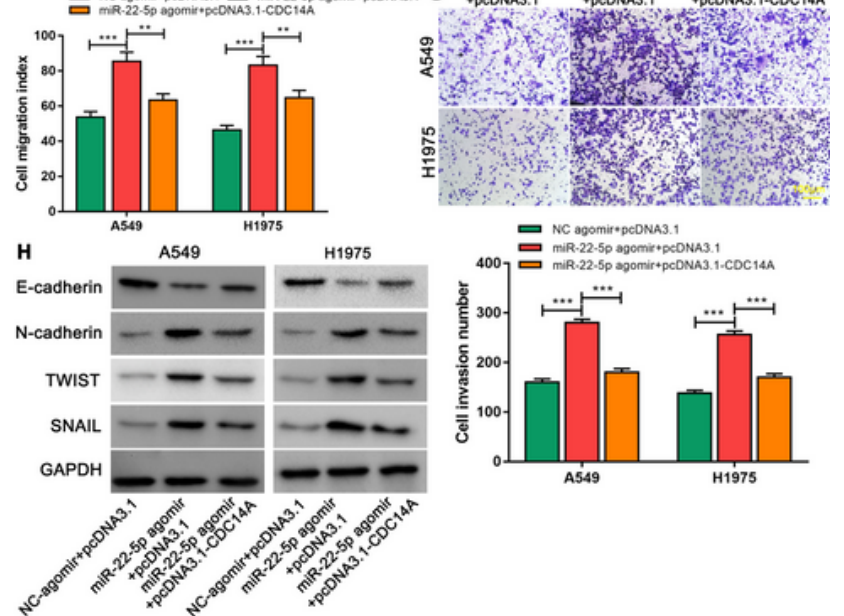

NC agomirppDDNA3.1

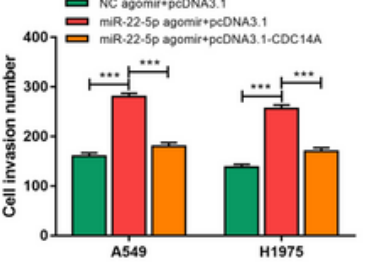

Figure 5 
MiR-22-5p promoted proliferation, migration and invasion of LUAD cells through targeting CDC14A. (A) The relative CDC14A mRNA level in LUAD tissues and adjacent non-tumor tissues were measured using quantitative real-time PCR. (B) The binding site of miR-22-5p on CDC14A was shown, and their interaction was validated using luciferase reporter assay. (C) The relative mRNA level of CDC14A after miR-22-5p/NC agomir was determined using quantitative real-time PCR. A549 and $\mathrm{H} 1975$ cells were co-transfected with miR-22-5p/NC agomir and pcDNA3.1-CDC14A/pcDNA3.1. (D) Cell counting kit-8 assay was performed to evaluate cell proliferation. (E) Cell cycle was detected using flow cytometry. (F) Wound healing assay was performed to evaluate cell migration. (G) Transwell assay was carried out to assess cell invasion. (H) The protein levels of E-cadherin, N-cadherin, TWIST and SNAIL was determined using western blotting.LINC00968: long intergenic non-protein coding RNA 968; LUAD: lung adenocarcinoma; CDC14A: cell division cycle 14A; NC: non-specific control; ${ }^{*} p<0.05,{ }^{* \star} p<0.01,{ }^{* \star \star} p<0.001$. 